\title{
CINCO NUEVAS ESPECIES DE ÁCAROS ORIBÁTIDOS (ACARI, ORIBATIDA) DE PINARES INCENDIADOS DE LA SIERRA DE GREDOS (ÁVILA, ESPAÑA) ${ }^{1}$
}

\author{
J. Gil-Martín * y L. S. Subías *
}

\begin{abstract}
RESUMEN
En este trabajo se describen cinco nuevas especies de Ácaros Oribátidos, encontradas en muestras colectadas en suelos de pinares incendiados de varias localidades de la Sierra de Gredos (Provincia de Ávila, Centro de España): Brachychthonius amicabilis n. sp., Hermanniella issanielloides n. sp., Ctenobelba pulchellula n. sp., Xenillus arilloi $\mathrm{n}$. sp., y Carabodes gregorioi n. sp.

Palabras Clave: Acari, Oribatida, Taxonomía, Pinares incendiados, Sierra de Gredos, España.
\end{abstract}

\section{ABSTRACT \\ Five new oribatid mite species (Acari, Oribatida) from burned pine forests in the Sierra de Gredos (Avila, Spain)}

Five new species of Oribatid mites found in soil samples collected in the burned pine forests of several localities in the Sierra de Gredos (Province of Avila, Central Spain) are described: Brachychthonius amicabilis n. sp., Hermanniella issanielloides n. sp., Ctenobelba pulchellula n. sp., Xenillus arilloi n. sp., and Carabodes gregorioi n. sp.

Key Words: Acari, Oribatida, Taxonomy, Burned pine forests, Sierra de Gredos, Spain.

\section{Introducción}

Las cinco especies de Ácaros Oribátidos nuevas para la Ciencia que se describen en el presente trabajo, pertenecen, una a la familia Brachychthoniidae Thor, 1934 del grupo de los Oribátidos Inferiores, Brachychthonius amicabilis n. sp., y las otras cuatro, del grupo de los Oribátidos Superiores Picnonóticos, una a la familia Hermanniellidae Grandjean, 1934, Hermanniella issanielloides n. sp., otra a la familia Ctenobelbidae Grandjean, 1965, Ctenobelba pulchellula $\mathrm{n}$. sp., otra más a la familia Xenillidae Woolley y Higgins, 1966, Xenillus arilloi n. sp., y una última a la familia Carabodidae C.L. Koch, 1837, Carabodes gregorioi n. sp.
Estas cinco nuevas especies, junto con otras dos que resultaron además ser nuevo género y se describen en trabajos aparte, han aparecido en el muestreo llevado a cabo en el verano del año 1992, para el estudio de los ácaros oribátidos de pinares incendiados de un sector de la cara sur de la Sierra de Gredos (Ávila) (Gil-Martín y Subías, in litt.).

\section{Material y Métodos}

Todas las localidades en que se han encontrado los ejemplares de las series típicas de las nuevas especies aquí descritas, pertenecen al muestreo realizado para el estudio antes referido, y los códigos

* Dpto. Biología Animal I. Facultad de Biología. Universidad Complutense. E-28040 Madrid. (e-mail: subias@eucmax.sim.ucm.es). 1 Investigación financiada por la Comisión Interministerial de Ciencia y Tecnología -CICYT- (Proyecto FOR91-1091-CO2-02) y por la Institución de Investigaciones y Estudios Abulenses "Gran Duque de Alba". 
con que aparecen son los utilizados en ese estudio global del que el presente trabajo es resultado parcial (ver Iturrondobeitia y Gil-Martín, in litt.).

En la descripción de las especies, se han seguido la caracterización y la terminología, sobre los rasgos morfológicos del cuerpo de los Oribátidos, ofrecidas por Pérez-Íñigo (1969), Balogh (1972), Subías (1978) y Mahunka y Zombori (1985).

Todo el material estudiado -serie típica-, se encuentra conservado en tubos con ácido láctico al $70 \%$ o en preparaciones semipermanentes con líquido de Hoyer, y depositado en el Departamento de Biología Animal I (Cátedra de Entomología) de la Facultad de Biología de la Universidad Complutense de Madrid.

Las derivatio nominis de todas las especies descritas en el presente trabajo, han sido realizadas por el primer autor.

\section{Resultados y Discusión}

\section{Brachychthonius amicabilis n. sp. (Fig. 1)}

MATERIAL TIPO: Se cuenta con tres ejemplares de esta especie, uno de ellos designado holotipo y dos paratipos, conservados en ácido láctico al $70 \%$. Fueron colectados en las localidades y muestras que a continuación se indican: uno en P1CS (Arenas de San Pedro 1, centro, superficie), uno en G2CS (Guisando 2, centro, superficie) y uno en G1CP (Guisando 1, centro, profundidad).

DESCRIPCIÓN: Dimensiones y tegumento: Longitud: 150-188 $\mu \mathrm{m}$; anchura: 75-81 $\mu \mathrm{m}$. Tegumento de color blanquecino, debido a un muy débil esclerosamiento de su cutícula. Ornamentación prácticamente inexistente tanto en el prodorso como en el primer segmento notogastral $(C D)$, y sencilla y poco marcada en los dos segmentos restantes $(E \mathrm{y}$ $P Y)$, donde se presenta prácticamente reducida a sendas líneas longitudinales latero-mediales, que distinguen tres porciones en el segmento, dos laterales y una media -típico en todo el grupo-.

Prodorso (Fig. 1a): Borde del rostro finamente aserrado. Setas en general todas lisas y ocupando su posición habitual. Setas rostrales de mayor tamaño, setas lamelares, interlamelares y exobotrídicas anteriores pequeñas y curvadas, y setas exobotrídicas posteriores ausentes.

Botridio típico de toda la familia Brachychthoniidae y sensilo, también de forma habitual, fusiforme con la cabeza cubierta de varias hileras de bárbulas o cortos pelos.

Notogáster (Fig. 1a): Setas notogastrales en número y posición habituales, en general, muy cor-
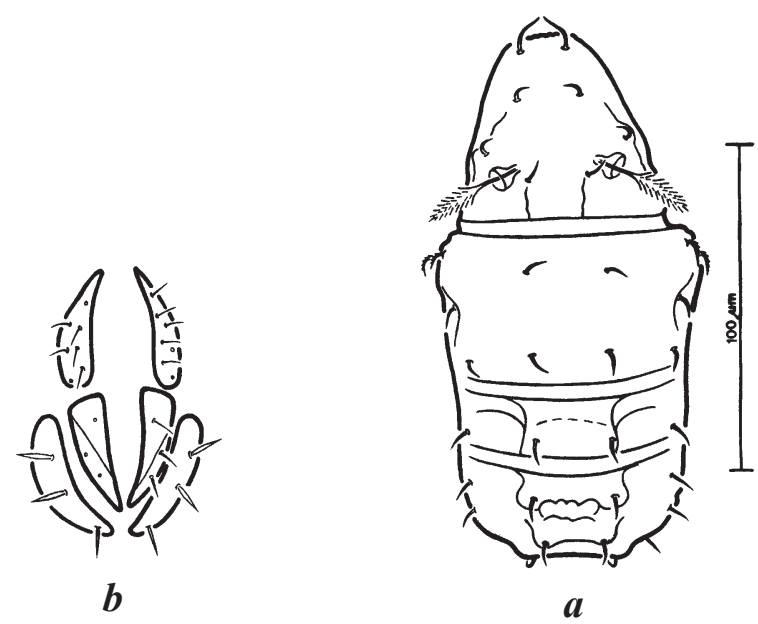

Fig. 1.-Brachychthonius amicabilis n. sp. (a) Visión dorsal, (b) Región genito-anal.

Fig. 1.-Brachychthonius amicabilis n. sp. (a) Dorsal view, (b) Genito-anal region.

tas y lisas, excepto las $c_{2}$ y $c_{3}$ que están ligeramente barbuladas por su borde externo.

Caracteres laterales: Se corrobora en esta posición, tanto la barbulación de las setas notogastrales $c_{2}$ y $c_{3}$ como la inexistencia de las setas exobotrídicas posteriores (exp), aunque si está presente el alvéolo.

Se observa, como es característico, la placa suprapleural $S p E$, y no se aprecia que la seta $c_{3}$ esté implantada sobre la placa suprapleural $S p C$.

Región genito-anal (Fig. 1b): Placas genitales con los siete pares habituales de setas, cortas, lisas y muy finas. No se han observado ni placas ni setas adgenitales.

Placas preanales y anales con uno y dos pares de setas, respectivamente, también cortas, lisas y muy finas. Placas adanales con tres pares de setas más robustas que las anteriores, siendo las $a d_{2}$ y $a d_{3}$ más gruesas que las $a d_{l}$.

Las setas que no se han dibujado -preanal, anales y algunas genitales, y la notogastral $p s_{2^{-}}$, ha sido por mantener la fidelidad al ejemplar designado como holotipo, si bien éstas si se hallan presentes en la especie y ofrecen las mismas características que sus semejantes, según se constata con la observación de los otros ejemplares de la serie típica.

Discusión: Realizada la exhaustiva revisión de los taxa actualmente descritos dentro del género, y 
consultando de un modo especial una de las más recientes y completas obras de revisión de la familia, en general, y del género, en particular (Moritz, 1976), se puede concluir que los ejemplares estudiados -que se propone corresponden a una nueva especie- no se parecen a ninguno de los allí descritos, asemejándose únicamente a los de la especie Brachychthonius pseudoimmaculatus descrita posteriormente por Subías y Gil (1991) a partir de muestras del Cabo de San Vicente (Portugal).

La nueva especie se diferencia de la referida como semejante, en que: (1) presenta la escultura del prodorso y primer segmento del notogáster muy tenue o prácticamente inexistente, y con dibujos apenas definidos en los segmentos segunto y tercero del notogáster, y (2) presenta las setas notogastrales $c_{2}$ y $c_{3}$ ligeramente barbuladas -la otra sólo muestra bárbulas en la última-.

DERIVATIO NOMINIS: El nombre específico de este nuevo taxón, se dedica: (1) a uno de los valores supremos que rigen las relaciones entre los seres humanos, la amistad, y (2) a los seres en quienes ese valor toma cuerpo y significado, los amigos.

\section{Hermanniella issanielloides n. sp. (Fig. 2)}

MATERIAL TIPO: Se cuenta con ocho ejemplares de esta especie, uno ha sido designado holotipo y el resto son los paratipos, todos ellos conservados en ácido lactico al70 \%. Fueron colectados en las localidades y muestras que a continuación se indican: dos en G1CS (Guisando 1, centro, superficie) y seis en G1CP (Guisando 1, centro, profundidad).

DESCRIPCIÓN: Dimensiones y tegumento: Longitud: 544-613 $\mu \mathrm{m}$; anchura: 325-350 $\mu \mathrm{m}$. Coloración general pardo oscura, habitual en el género, y "aspecto" general parecido a una "Issaniella".

Prodorso (Fig. 2a): Superficie del prodorso ligeramente granulada, y en su parte anterior, desde la altura de los botridios hasta el rostro, con depresiones o fosetas abiertas.

Rostro de forma redondeada, y setas rostrales de gran tamaño, barbuladas, ligeramente recurvadas hacia el eje del cuerpo e insertas en ambos márgenes laterales del rostro. Setas lamelares de tamaño semejante a las rostrales, barbuladas e insertas sobre unos patentes salientes laterales, visibles dorsalmente. Setas interlamelares ligeramente más robustas que las anteriores e igualmente barbuladas, y que se insertan sobre una visible línea interbotrídica determinada por un marcado cambio de pendiente.
Botridios grandes, prominentes y en forma de copa, y sensilos de una longitud aproximadamente la mitad que una seta interlamelar, con un tallo liso y una cabeza fusiforme, estrecha y cubierta por varias filas de finos cilios. Setas exobotrídicas situadas por detrás y cerca del botridio, muy cortas -espiniformes- y no visibles dorsalmente.

Notogáster (Fig. 2a): La exuvia tritoninfal que lo cubre presenta una escultura constituida por una masa regular de montículos y depresiones que se hace más evidente en las zonas laterales donde se ven de perfil, y porta diez pares de setas -en su posición habitual- robustas, espiniformes y barbuladas, destacando el último par, más largo, erecto y dirigido hacia atrás. Fisuras im bien visibles y situadas en su posición habitual. Los cinco pares de setas notogastrales posteromarginales se explican desde donde mejor se observan, por el lado ventral.

Lado ventral (Fig. 2b): Setas posteromarginales del notogáster insertas de un modo ligeramente ventral, de tamaño semejante entre si -aproximadamente la mitad que las setas exuviales- y con un tallo o eje central densamente ciliado por ambos bordes.

Toda la region ventral presenta una cutícula con salientes unidos unos a otros formando hileras de aspecto "aserrado". Setas coxiesternales pequeñas y densamente barbuladas, y dispuestas siguiendo la formula 3-1-3-2. Placas genitales con siete pares de setas (en el ejemplar dibujado la derecha sólo presenta seis y no se aprecia el alveolo de la séptima) semejantes a las epimerales, aunque algo más pequeñas. Se observa un par de setas adgenitales semejantes a las coxiesternales. Las placas anales presentan la superficie con líneas de diseño algo menos dentado que la placa ventral, y portan dos pares de setas semejantes a las posteromarginales (y adanales), pero más estilizadas que éstas. Se observan los tres pares de setas adanales en su posición habitual. Las fisuras adanales (iad) se encuentran en posición transversal al eje del cuerpo y a la altura del borde anterior del orificio anal.

Patas: Los tarsos de las patas son todos monodáctilos.

DISCUSIÓN: Su parecido a Issaniella Grandjean, 1962, mencionado con anterioridad, viene de su escultura notogastral, y del gran desarrollo y abertura lateroanterior del botridio.

Esta especie se diferencia bien de la mayoría de las hasta ahora descritas en el género por: (1) sus modestas dimensiones -dentro del género- (544$612 \times 325-350 \mu \mathrm{m}$ ), (2) su escultura notogastral (con tubérculos y valles, regulares en forma y homogéneamente distribuidos), (3) su sensilo de 

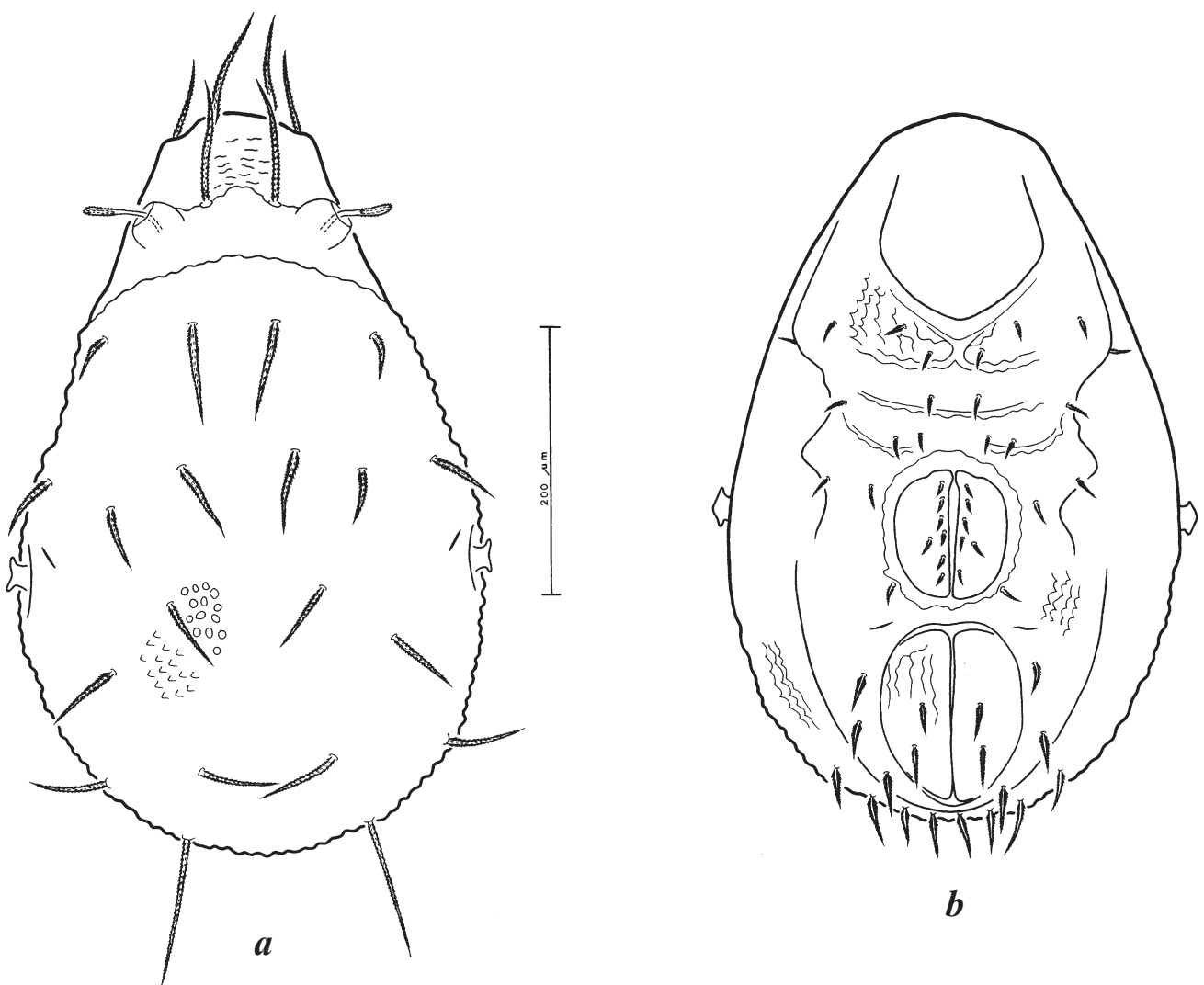

b

Fig. 2.- Hermanniella issanielloides n. sp. (a) Visión dorsal, (b) Visión ventral.

Fig. 2.- Hermanniella issanielloides n. sp. (a) Dorsal view, (b) Ventral view.

tallo relativamente corto y cabeza muy barbulada, y (4) sus setas del lado ventral barbuladas.

Concretando, por la forma del sensilo se acercaría a las especies Hermanniella granulata (Nicolet, 1855) y Hermanniella incondita Pérez-Iñigo, 1987, pero la primera especie es de un tamaño medio algo superior y la segunda muy superior, y ambas presentan muy diferente escultura notogastral; observándose asimismo diferencias en la características de las setas de todo el lado ventral.

Por las dimensiones y la ornamentación notogastral, mediante tubérculos, se acercaría a las especies Hermanniella mastyx Mahunka, 1983 y Hermanniella clavasetosa Hammer, 1966, diferenciándose de ambas principalmente por la forma del sensilo y la longitud y forma de las setas notogastrales. No obstante las diferencias expuestas con respecto a estas dos últimas especies, debe hallarse, por su aspecto general, más próxima a ellas que a las que se ofrecieron en la primera comparación, si bien $H$. mastyx y $H$. clavasetosa fueron descritas de latitudes muy distantes a donde apareció la nueva especie: Tanzania (Mahunka, 1983) y Nueva Zelanda (Hammer, 1966), respectivamente.

DERIVATIO NOMINIS: El nombre específico otorgado a este nuevo taxón, refleja el gran parecido en el aspecto general (que nos llevó a dudar inicialmente de su ubicación taxonómica), que presentan estos ejemplares con los de especies ubicadas dentro del género Issaniella.

Ctenobelba pulchellula n. sp. (Fig. 3)

Material tiPo: Se cuenta con un único ejemplar de esta especie, el holotipo, que se encuentra conservado en ácido láctico al $70 \%$, y que fue colectado en la localidad y muestra V1CP (Cuevas del Valle 1, centro, profundidad).

DESCRIPCIÓN: Dimensiones y tegumento: Longitud: $456 \mu \mathrm{m}$; anchura: $238 \mu \mathrm{m}$. Coloración entre amari- 

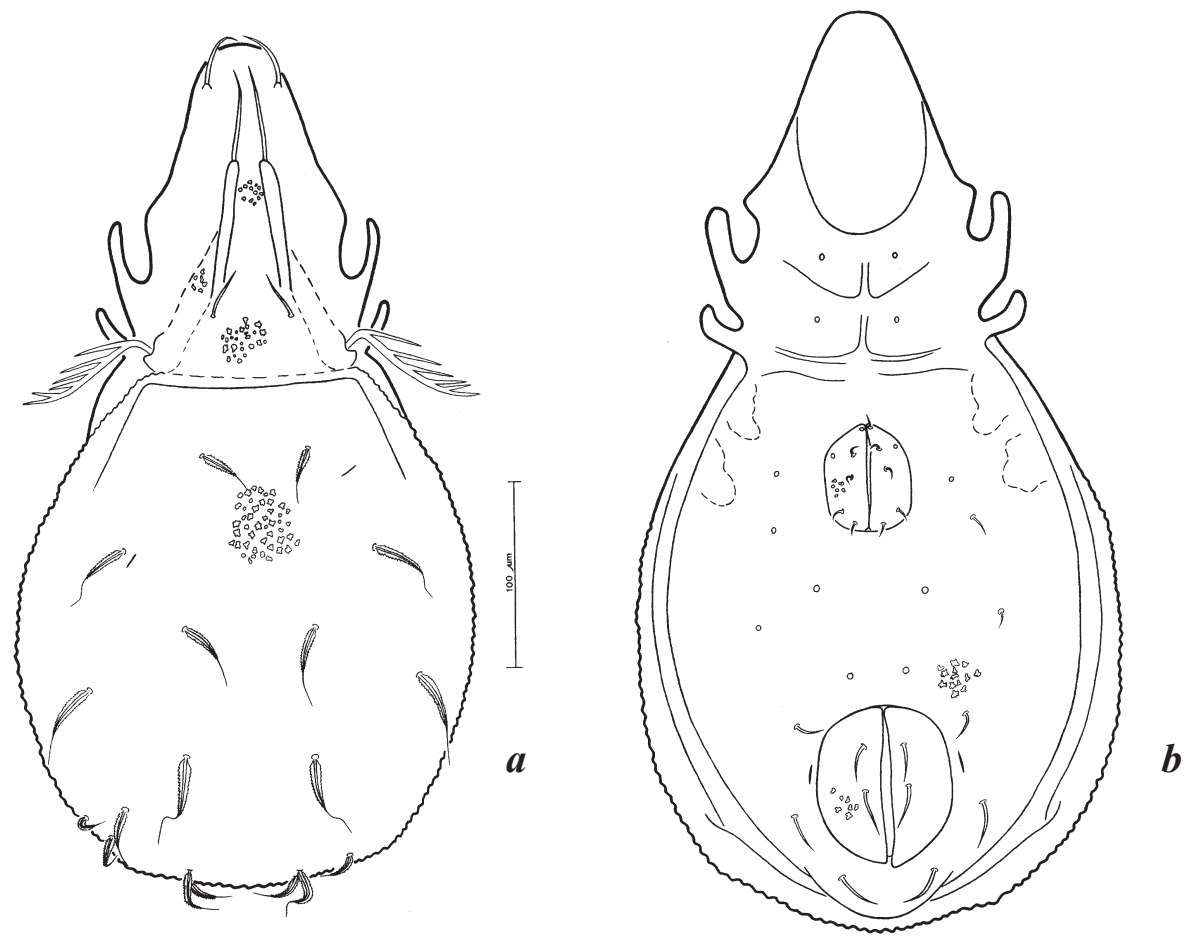

Fig. 3.-Ctenobelba pulchellula n. sp. (a) Visión dorsal, (b) Visión ventral.

Fig. 3.-Ctenobelba pulchellula n. sp. (a) Dorsal view, (b) Ventral view.

llo ocráceo y marrón claro, y ornamentación general cerotegumentaria de toda la superfice del cuerpo -incluidas las placas genital y anal en el lado ventral- determinada por un denso granulado en forma de estrella, irregulares en la forma de su borde y en tamaño.

Prodorso (Fig. 3a): Rostro redondeado, y lamelas bien marcadas, prácticamente rectas, bastante próximas entre si y de disposición ligermente convergente hacia el extremo distal -que es redondeado-. Setas rotrales insertas dorsalmente, de buen tamaño, lisas y curvadas hacia el eje del cuerpo; setas lamelares insertas en el extremo distal de las cóstulas lamelares, dirigidas hacia adelante, de tamaño semejante a las rostrales -no llegan al borde del rostro-, lisas y prácticamente rectilíneas; y setas interlamelares insertas al comienzo de las cóstulas lamelares, de tamaño la mitad que las lamelares y también lisas.

Botridio con escama o espolón posterior, y sensilo pectinado habitual, con un tallo largo y de final puntiagudo, y cinco ramas laterales de longitud decreciente desde la base hacia el extremo.

Notogáster (Fig. 3a): Borde anterior casi rectilíneo y sólo ligeramente cóncavo hacia el prodorso, con dos líneas sobre la superficie que determinan sendas ligeras prominencias dorsales a modo de "hombros" a la altura de los botridios. Todo el reborde exterior del notogáster, presenta un aspecto aserrado, que se corresponde con la visión lateral del granulado de la superficie. Diez pares de setas notogastrales -aunque en el lado derecho en el holotipo dos han desaparecido, probablemente las $p_{3} \mathrm{y}$ $h_{2^{-}}$, bien patentes, con un tallo central que acaba en un flagelo a veces en forma de "látigo" y un amplio reborde hialino aserrado presente desde su base en la mitad o tres cuartas partes de la seta. Las setas posteriores curvadas hacia el lado ventral. Fisuras im, la del lado izquierdo en su posición normal y la del lado derecho muy por delante de la seta $l a$.

Lado ventral (Fig. 3b): No se han observado varias de las setas coxiesternales, probablemente debido a que el ejemplar base de esta descripción se encuentra recubierto en esa zona de restos de materiales del suelo, aunque quizá también pueda haberse depilado parcialmente en alguna fase del proceso de manipulación. Los epímeros están bien marcados. La ornamentación de la región ventral es la descrita para toda la superficie del animal, y presentándose también en las placas genitales y anales. 

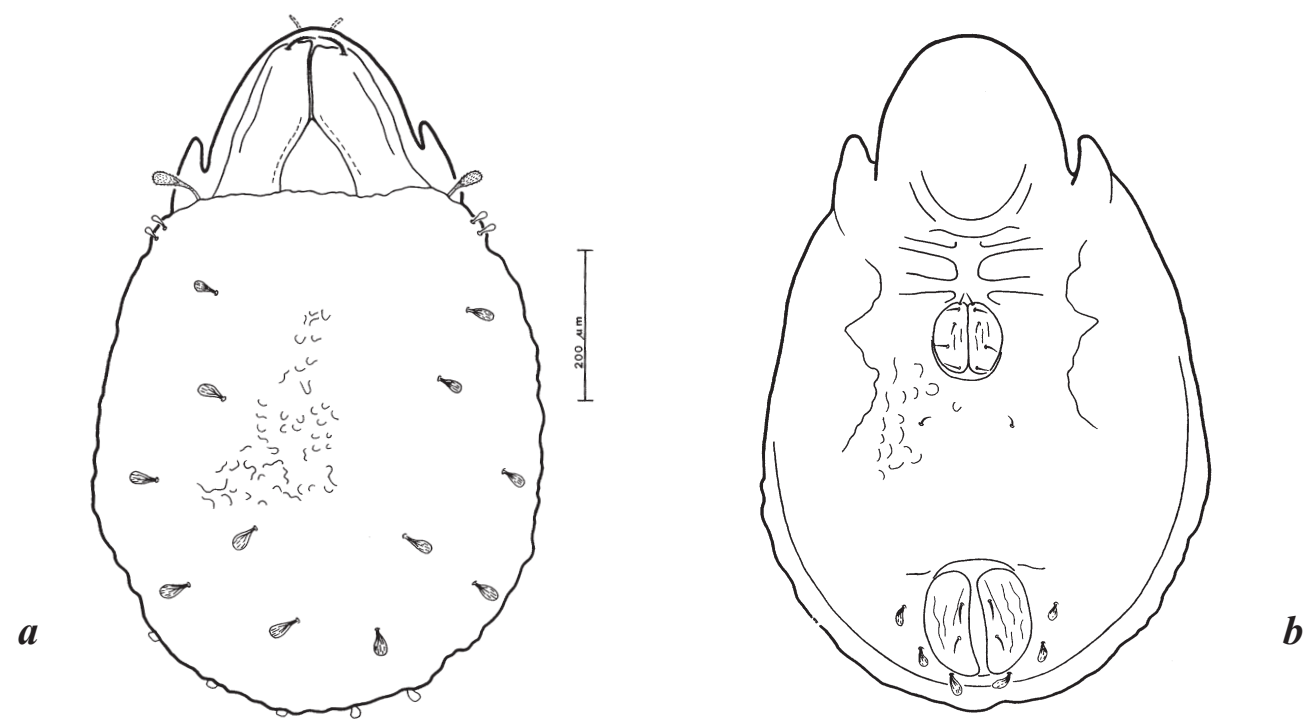

Fig. 4.- Xenillus arilloi n. sp. (a) Visión dorsal, (b) Visión ventral.

Fig. 4.-Xenillus arilloi n. sp. (a) Dorsal view, (b) Ventral view.

Placas genitales de un tamaño la mitad que la anales y portando seis pares de setas lisas y muy pequeñas. Setas adgenitales, tres pares, lisas y de tamaño algo mayor que las genitales (en la mayor parte sólo observable el alveolo, por desprendimiento de la seta). Placas anales con dos pares de setas grandes y lisas, y zona adanal con tres pares de setas semejantes a las anales, y con fisuras iad en situación y posición habituales, entre la setas $a d_{2}$ y $a d_{3}$ y paralelas al borde de la placa.

Patas: Todos los tarsos son monodáctilos.

Discusión: Esta especie presenta semejanzas con Ctenobelba foliata Hammer, 1961 y con Ctenobelba parafoliata Pérez-Iñigo Jr., 1991, ambas especies de dimensiones parecidas y con setas notogastrales con tallo, ensanchamiento hialino -en forma de hoja- y flagelo distal.

Se diferencia esta especie de C. foliata, porque: (1) las setas notogastrales $c_{2}$ y $l m$ no son significativamente más pequeñas que el resto, (2) el borde externo del ensanchamiento hialino de las setas notogastrales es claramente aserrado, (3) el estrellado que significa la ornamentación general del cerotegumento también se halla presente en el prodorso, y (4) las setas interlamelares son de una longitud la mitad que las lamelares. Ni en la descripción original de la especie (Hammer, 1961) ni en subsiguientes redescripciones, se hace la caracteri- zación del lado ventral, por lo que no se puede contrastar desde esta perspectiva.

Se diferencia esta especie de $C$. parafoliata, porque: (1) el extremo distal de las setas notogastrales acaba en un largo, y a veces recurvado, flagelo, (2) presenta un característico estrellado cuticular como ornamentación general tanto dorsal como ventral, (3) las cóstulas lamelares están más juntas, (4) las setas interlamelares son lisas y más cortas que las lamelares, y (5) el sensilo presenta cinco ramas laterales. Además nuestro holotipo, presenta unas dimensiones algo inferiores al mínimo ofrecido por PérezÍñigo Jr. (1991) en la descripción de C. parafoliata.

DERIVATIO NOMINIS: Nombre específico dedicado a $\mathrm{M}^{\mathrm{a}}$ Isabel Menéndez, esa persona "encantadora" que con su existencia alienta mi vida.

\section{Xenillus arilloi n. sp. (Fig. 4)}

Material tiPo: Se cuenta con dos ejemplares de este especie, uno de ellos designado holotipo y un paratipo, conservados en ácido láctico al 70\%, y colectados en la localidad y muestra E1PS (San Esteban del Valle 1, periferia, superficie).

DESCRIPCIÓN: Dimensiones y tegumento: Longitud: 775-1.000 $\mu \mathrm{m}$; anchura: 488-625 $\mu \mathrm{m}$. Coloración del cuerpo, pardo oscuro. 
Prodorso (Fig. 4a): Rostro amplio y redondeado, en cuyo borde se insertan, aunque se observan con dificultad, las setas rostrales pequeñas y recuvadas hacia el lado ventral

Lamelas anchas cubriendo gran parte de la superficie del prodorso, llegando casi al rostro, con su borde externo muy cercano y paralelo al borde exterior del prodorso y sus bordes internos fusionados en la base y muy próximos entre si a lo largo de la mitad anterior de su recorrido. Presentan sendas líneas bien patentes cercanas al margen externo, y el extremo de la cúspide lamelar truncado o ligeramente cóncavo, con el ángulo interno en forma de diente visible aunque muy poco marcado y el ángulo externo completamente redondeado. Setas lamelares insertan sobre el ángulo externo de la cúspide lamelar, fuertemente curvadas hacia el eje del cuerpo, de modesto tamaño y finísimamente barbuladas. No se han observado setas ni alveolos en la región interlamelar. Botridios totalmente tapados por el borde anterior del notogáster, con sensilos fusiformes formados por un tallo corto y un ensanchamiento distal mazudo, todo él recubierto de hileras de finas bárbulas. En la superfice del prodorso no se hace evidente ningún tipo de relieve u ornamentación. No se ha apreciado la existencia de mucrón entre las lamelas, pero la superfie del prodorso no se observa del todo nítida por lo que no se puede afirmar que carezca de él.

Notogáster (Fig. 4a): Forma general, notablemente ancho; su borde anterior es prácticamente recto y el aspecto de todo su borde libre exterior es rugoso, como corresponde a la visión lateral de la fuerte ornamentación notogastral. En efecto, la ornamentación de la superficie del notogáster se corresponde con un relieve rugoso constituido por un "laberinto de canales y crestas" de diseño o distribución muy irregular. Setas notogastrales en número habitual, 11 pares -si bien en el lado derecho se observan sólo diez, faltando la seta $p_{3^{-}}$, de tamaño medio-grande y con forma de raqueta. Los dos pares anteriores de menor tamaño y situadas en la región humeral insertas a poca distancia del borde notogastral, y los tres pares posteriores insertos sobre el mismo borde posterior del notogáster. No se observan la fisuras de esta región del cuerpo debido a la ornamentación tegumentaria notogastral fuertemente rugosa.

Lado ventral (Fig. 4b): La ornamentación de toda la placa ventral es muy semejante a la del notogáster, estando constituida en las placas genitales y anales por una serie de líneas longitudinales. Los apodemas epimerales se encuentran bien marcados, pero no se han encontrado setas en toda la región epimeral. Setas genitales, cinco pares, setiformes, pequeñas y lisas; setas adgenitales, un par, semejantes a las genitales y recurvadas en su extremo; setas anales, dos pares, mayores que las genitales y también lisas, y setas adanales, tres pares, de forma semejante a las notogatrales, de mayor tamaño las $a d_{1}$ y más pequeñas las $a d_{2}$ y $a d_{3}$. Las fisuras iad curvadas, situadas a la altura del ángulo anterior de la abertura genital y en posición transversal al eje del cuerpo y a la línea de apertura de las placas anales.

Patas: Los fémures presentan quillas ventrales, y todos los tarsos son tridáctilos.

Discusión: Esta especie presenta cierta similitud general, en la ornamentación del notogáster y en la forma del sensilo y de las setas notogastrales, así como en las dimensiones dadas para la especie por Arribas et al. (1984), con Xenillus clavatopilus Mihelcic, 1966, si bien se diferencia de ella abiertamente por: (1) la forma de las cúspides lamelares, aquí completamente romas -más parecido en las lamelas a Xenillus clypeator Robineau-Desvoidy, 1839-, (2) la posición, muy extraña a nivel genérico, de las fisuras iad, a la altura del ángulo anterior de las placas anales perpendiculares al eje sagital/logitudinal del cuerpo, y (3) la forma de las setas anales, aquí setiformes. Además, las setas notogastrales, si bien las de la referida especie son las más semejantes por su aspecto general, son aquí más cortas y ensanchadas.

También se asemeja esta especie a Xenillus penicilliger Csiszár, 1961, por los mismos caracteres en que se acercaba a la anteriormente comparada, siendo caracteres diferenciales de la nueva especie propuesta: (1) la irregularidad de las formas que dibujan la ornamentación notogastral, lejos de las regulares foveolas de la especie comparada, y (2) la forma de las setas lamelares, no ensanchadas en su extremo. Además el tamaño de las setas notogastrales parece menor en $X$. penicilliger. Las características ventrales no se pueden contrastar convenientemente, ya que Csiszár (1961) no ofrece sino una brevísima reseña de esta visión, sin dibujo desde esa perspectiva.

Si ciertamente la especie descrita no presenta mucrón, que como se ha dicho no se ha podido observar, se diferenciaría también por ello de las especies contrastadas.

DERIVATIO NOMINIS: Dedicado a mi compañero el Dr. Antonio Arillo, por su ayuda en el intento de descifrar el contenido de este pequeño mundo y por su estimable contribución al conocimiento de los Oribátidos. 

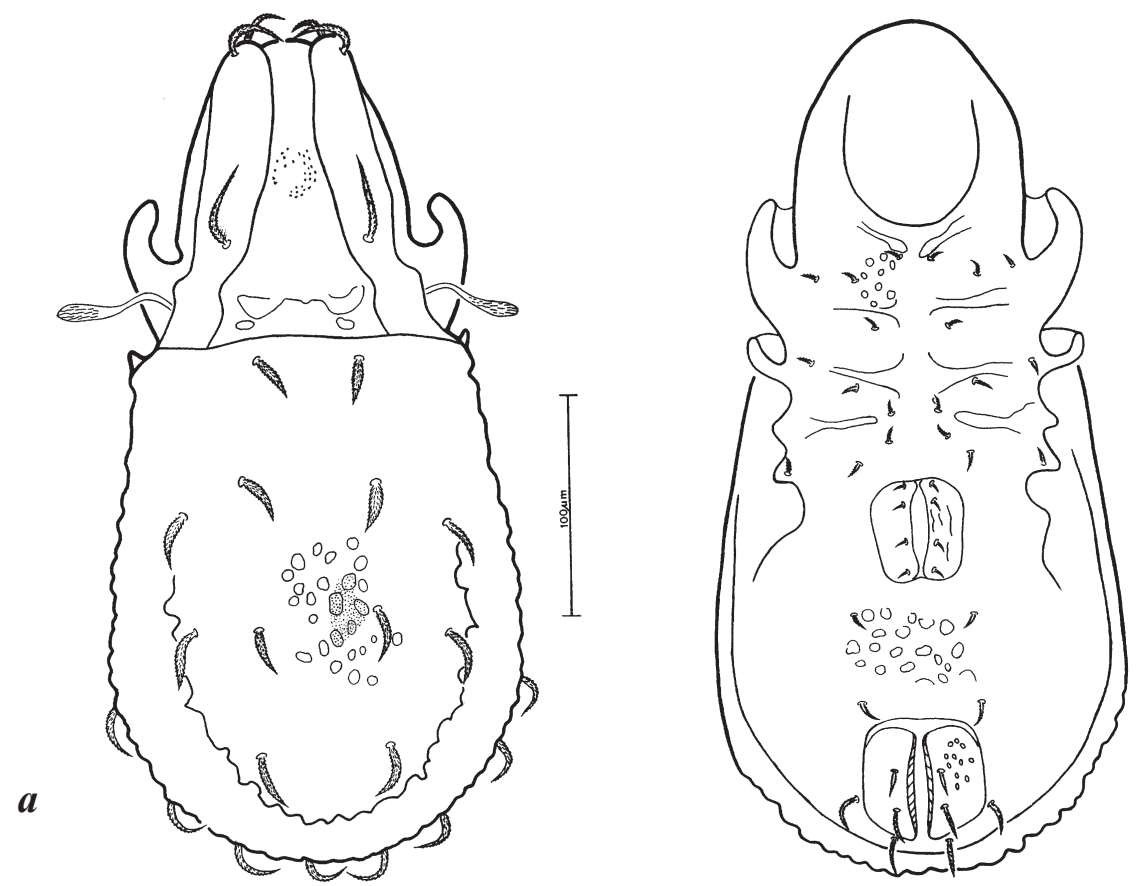

Fig. 5.-Carabodes gregorioi n. sp. (a) Visión dorsal, (b) Visión ventral.

Fig. 5.- Carabodes gregorioi n. sp. (a) Dorsal view, (b) Ventral view.

Carabodes gregorioi n. sp. (Fig. 5)

MATERIAL TIPO: Se dispone de cuarenta y tres ejemplares de esta especie, el holotipo y cuarenta paratipos conservados en ácido acético al 70\%, y dos paratipos montados en preparación semipermanente con líquido de Hoyer. Fueron colectados en las localidades y muestras que a continuación se indican: tres en G3PP (Guisando 3, periferia, profundidad), siete en P1CS (Arenas de San Pedro 1, centro, superficie), nueve en P1CP (Arenas de San Pedro 1, centro, profundidad), uno en P1PP (Arenas de San Pedro 1, periferia, profundidad), dos en $\mathrm{H} 2 \mathrm{CP}$ (El Hornillo 2, centro, profundidad), uno en G4PS (Guisando 4, periferia, superficie), ocho en C1CP (Candeleda 1, centro, profundidad) y doce en C1PP (Candeleda 1, periferia, profundidad).

DESCRIPCIÓN: Dimensiones y tegumento: Longitud: valores extremos de 213 y $413 \mu \mathrm{m}$, y media de 362 $\mu \mathrm{m}$; anchura: valores extremos de 150 y $200 \mu \mathrm{m}, \mathrm{y}$ media de $169 \mu \mathrm{m}$. Coloración marrón oscuro y aspecto general parecido a un Odontecepheus.

Prodorso (Fig. 5a): Rostro redondeado, y lamelas largas -sobrepasando ligeramente el límite del rostro-, anchas y ligeramente convergentes hacia el extremo distal. Setas interlamelares insertas en la parte central de la zona basal de la lamela -algo por delante de la altura botridio-, largas, robustas, recu- biertas de varias hileras de cortos cilios y ligeramente recurvadas hacia el eje de cuerpo; setas lamelares insertas en el ángulo externo de la cúspide lamelar ligeramente por dentro del borde, de tamaño y aspecto semejante a las interlamelares y fuertemente recurvadas hacia el eje del cuerpo; y setas rostrales de características semejantes a las lamelares y de un tamaño ligeramente inferior.

La superficie del prodorso se halla cubierta de un denso punteado, y en su zona basal -cerca de la sutura disyugal- con un par de áreas claras de diseño circular. Sensilo fusiforme, formado por un largo tallo y una cabeza, que resulta un engrosamiento distal del tallo sin solución de continuidad, cubierta de unas líneas o cilios, que en el reborde apical se manifiestan como una rugosidad ligerísima y apenas perceptible.

Notogáster (Fig. 5a): Borde anterior rectilíneo, tendiendo a ligeramente convexo. Presenta dos pequeños y agudos salientes humerales dirigidos hacia adelante. La ornamentación de su cutícula, fuertemente esclerosada y punteada en toda su superficie, se compone de una densa masa de patentes foveolas de forma redondeada, aunque heterogéneas en forma y tamaño. Se observa una 
línea, de borde sinuoso -debido al foveolado dorsal, que viene determinada por un acusado cambio de pendiente que discurre paralela al borde exterior del notogáster, y cercana y por fuera de la inserción de las setas $l a, h_{2}$ y $h_{1}$. Presenta diez pares de setas notogastrales en su posición habitual, más robustas que las prodorsales -algo más cortas y gruesas-, igualmente recubiertas de hileras de cortos cilios, y casi todas recurvadas hacia el eje de cuerpo.

Un ejemplar de la serie típica de esta nueva especie presentaba lo que consideramos debe corresponder a una anomalía quetotáxica en la posición $c_{2}$, donde aparecían partiendo del mismo alveolo dos setas del mismo tamaño y semejantes a las descritas en el holotipo.

Lado ventral (Fig. 5b): Toda la placa ventral se halla recubierta de un foveolado semejante al dorsal pero algo más tenue, siendo éstas foveolas de menor tamaño en las placas anales y presentando como excepción simplemente un ligero estriado en la placas genitales. Se observan bien marcados todos los apodemas en la región epimeral, y la fórmula quetotáxica habitual 3-1-3-3, siendo estas setas coxiesternales pequeñas y barbuladas. Setas genitales, cuatro pares, pequeñas y lisas; setas adgenitales, un par, semejantes a las epimerales; setas anales, dos pares, mayores que las epimerales y genitales y fínamente barbuladas, más pequeñas las del par anterior que las del posterior; y setas adanales, tres pares, de tamaño y carcterísticas semejantes a las anales, parecidas en tamaño las del par anterior de anales y las $a d_{3}$-que están en posición preanal- y las del par posterior de anales y las $a d_{1}$ y $a d_{2}$-que están en posición postanal-. Debido al fuerte esclerosamiento de su cutícula, no han podido ser observadas las fisuras iad (como no se puedieron observar dorsalmente la fisuras in).

Patas: Todos los tarsos son monodáctilos.

DisCUSIÓN: Consultadas y analizadas las numerosas descripciones originales de todas las especies del género, mayoritariamente reseñadas en la revisión que realiza Mahunka (1987) de la familia Carabodidae C.L. Koch, 1836, no se ha encontrado igualdad con ninguna de ellas, observándose únicamente semejanza con dos especies: Carabodes palmifer Berlese, 1904 y Carabodes areolatus Berlese, 1916, encuadrables dentro de un grupo caracterizado por tener el cuerpo con forma general alargada, el borde anterior del notogáster truncado o algo curvado, y las lamelas largas, con bordes bien marcados y paralelas.

Tanto la nueva especie como las dos referidas para contrastar, presentan un foveolado o areolado notogastral semejante, sin embargo mientras que en C. gregorioi n. sp. y C. palmifer el prodorso no presenta areolas, en $C$. areolatus si hay. Se ha tenido en cuenta a la hora de tener en consideración la ausencia de areolas en el prodorso de C. palmifer, la redescripción que de la especie de Berlese hace Bernini (1970), si bien este autor considera sinónimo suyo a Carabodes peniculatus Aoki, 1970, y sin embargo en la descripcion de ésta (Aoki, 1970) se aprecia el prodorso claramente areolado.

La nueve especie se diferencia de las dos anterires por tener: (1) un tamaño medio más pequeño 312,5 x $175 \mu \mathrm{m}$ - (Gil-Martín y Subías, op. cit.) , (2) setas notogastrales robustas, con el extremo en punta y densamente ciliadas, y (3) sensilo de tallo largo, fusiforme y con la cabeza finamente ciliada. En C. palmifer, de dimensines: 460 × $220 \mu \mathrm{m}$ según Berlese (1904), las setas notogastrales son mucho más cortas, y de cabeza roma y con el extremo ciliado, y el sensilo es asimismo muy corto. En $C$. areolatus, de dimensiones: 440 × $235 \mu \mathrm{m}$ según Berlese (1916), las setas notogastrales, también robustas, no acaban en punta, sino en una serie de espinas apicales a distinto nivel, y el sensilo presenta el extremo truncado y aserrado por largas espinas.

DERIVATIO NOMINIS: Dedicada a la persona que compartió conmigo los esfuerzos del muestreo en el monte, Gregorio Martín, por su generosidad y su desinteresada aportación en la ampliación de los horizontes de nuestro conocimiento.

\section{Referencias}

Aoki, J., 1970. The Oribatid Mites of the Islands of Tsushima. Bull. Nat. Sci. Mus. Tokyo, 13(3): 395-442.

Arribas, M.A., Subías, L.S. \& Ruiz, E., 1984. Oribátidos (Acarida, Oribatida) Superiores Gimnonóticos del "sabinar albar" español. Cuad. Invest. Biol. (Bilbao), 5: 57-63.

BAlogh, J., 1972. The oribatid genera of the world. Akadémiai Kiadó. Budapest. 189 pp.

Berlese, A., 1904. Acari nuovi. Manipulus IIus. Redia, 1: 258-280.

Berlese, A., 1916. Certuria terza di Acari nuovi. Redia, 12: 289-338.

Bernini, F., 1970. Notulae Oribatologicae II. Gli Oribatei (Acarida) delle Alpi Apuane ( $1^{\text {a }}$ serie). Lav. Soc. Ital. Biogeogr. (N.S.), 1: 390-429.

CsiszÁr, J., 1961. Neue Oribatiden (Acari). Folia Entomol. Hung., 14: 447-450. 
Gil-Martín, J. \& SubíAS, L.S., in litt. Estudio faunístico de los Oribátidos (Acari, Oribatida) de pinares incendiados de un sector de la cara Sur de la Sierra de Gredos (Avila). Bol. Asoc. Esp. Entomol.

ItUrRondobeitia, J.C. \& Gil-Martín, J., in litt. Estudio ecológico de los Oribátidos (Acari, Oribatida) de pinares incendiados de una zona de la Sierra de Gredos (Avila, España). 1. Análisis de la diversidad y el equilibrio de las poblaciones. Rev. Écol. Biol. Sol.

Hammer, M., 1961. A few new species of Oribatids from Southern Italy. Zool. Anz., 166: 113-119.

HAMMER, M., 1966. Investigations on the Oribatid fauna of New Zealand. Part I. Biol. Skr. Dan. Vid. Selsk., 15(2): 1-108.

MahunKa, S., 1964. Über die Gattung Ctenobelba Balogh, 1943 (Acari, Oribatei). Opusc. Zool. Budapest, 2: 223-228.

MahunKa, S., 1983. Oribatids from the eastern part of the Ethiopian Region (Acari). III. Acta Zool. Hung., 29(4): 397-440.

MahunKa, S., 1987. A survey of the family Carabodidae C. L. Koch, 1836 (Acari: Oribatida), II. Acta Zool. Hung., 33(3-4): 399-434.

MAHUnKA, S. \& ZomBori, L., 1985. The variability of some morphological features in Oribatid mites. Folia Entomol. Hung., 46(1): 115-128.
MorITZ, M., 1976. Revision der europäischen Gattungen und Arten der Familie Brachychthoniidae (Acari, Oribatei). Teil 2. Mixochthonius Niedbala, 1972, Neobrachychthonius nov. gen., Synchthonius v. d. Hammen, 1952, Poecilochthonius Balogh, 1943, Brachychthonius Berlese, 1910, Brachychochthonius Jacot, 1983. Mitt. Zool. Mus., 52(2): 227-319.

PÉrez-ÍÑ̃go, C., 1969. Acaros Oribátidos de suelos de España Peninsular e Islas Baleares ( $1^{\mathrm{a}}$ Parte). Graellsia, 24: 143-238.

PÉREZ-ÍÑ̃GO Jr., C., 1991. Oribátidos (Acari, Oribatei) de Menorca. Misc. Zool., 14: 29-40.

SuBíAS, L.S., 1978. Anomaloppia canariensis n. gen., n. sp. (Acarida, Oribatida, Oppiidae) de las Islas Canarias. Consideraciones filogenéticas sobre la familia. Redia, 61: 565-574.

SuBíAs, L.S. \& GiL, J., 1991. Tres nuevas especies de la familia Brachychthoniidae (Acari, Oribatida) del Sur de Portugal. Arq. Mus. Bocage (N.S.), 2(1): 1-10.

Recibido, el 2-IV-1997 Aceptado, el 2-VII-1997 Publicado, el 31-X-1997 